\title{
Application of the spirometer in respiratory gated radiotherapy
}

\author{
Tiezhi Zhanga) and Harry Keller \\ Department of Medical Physics, University of Wisconsin, Madison, Wisconsin 53706 \\ Matthew J. O'Brien \\ Department of Pulmonary Function, University of Wisconsin Hospital, Madison, Wisconsin 53792 \\ Thomas R. Mackie and Bhudatt Paliwal \\ Department of Medical Physics and Department of Radiation Oncology, University of Wisconsin, Madison, \\ Wisconsin 53792
}

(Received 23 May 2003; revised 9 September 2003; accepted for publication 23 September 2003; published 20 November 2003)

The signal from a spirometer is directly correlated with respiratory motion and is ideal for target respiratory motion tracking. However, its susceptibility to signal drift deters its application in radiotherapy. In this work, a few approaches are investigated to control spirometer signal drift for a Bernoulli-type spirometer. A method is presented for rapid daily calibration of the spirometer to obtain a flow sensitivity function. Daily calibration assures accurate airflow measurement and also reduces signal drift. Dynamic baseline adjustment further controls the signal drift. The accuracy of these techniques was studied and it was found that the spirometer is able to provide a long-term drift-free breathing signal. The tracking error is comprised of two components: calibration error and stochastic signal baseline variation error. The calibration error is very small $(1 \%$ of $3 l)$ and therefore negligible. The stochastic baseline variation error can be as large as $20 \%$ of the normal breathing amplitude. In view of these uncertainties, the applications of spirometers in treatment techniques that rely on breathing monitoring are discussed. Spirometer-based monitoring is noted most suitable for deep inspiration breath-hold but less important for free breathing gating techniques. (C) 2003 American Association of Physicists in Medicine. [DOI: 10.1118/1.1625439]

\section{INTRODUCTION}

Various respiratory motion control treatment techniques including gating, ${ }^{1-3}$ breath holding ${ }^{4}$ or active breathing control $(\mathrm{ABC})^{5,6}$ are used in radiotherapy to reduce the target margin due to respiratory motion. A reliable breathing monitoring system is needed for such techniques. The most commonly used systems can be classified into three categories:

(1) Measurement of the surface of the abdomen by tracking a reflective marker on the chest with a fixed camera ${ }^{2,7-10}$ or by measuring the distance from a fixed point to the surface of the abdomen using a laser-based distance sensor. ${ }^{1}$

(2) Tracking internal markers using $x$-ray ${ }^{11-15}$ or internal sensors using magnetic fields. ${ }^{16}$

(3) Measurement of the change in lung volume using a spirometer. $^{5,6}$

In this work, we will focus on the last category.

A spirometer measures airflow. The change in air volume is then inferred from the integrated airflow signal over time. Compared with displacement measurement-based systems, a spirometer has many advantages, including the following.

Better signal/target position correlation: The spirometer signal reflects lung volume changes, which is directly correlated with tumor position. Abdomen surface displacements do not always correlate with tumor position. Phase shift or delay $^{7,17}$ and pseudomotion (Fig. 1) have been observed.

Less setup variation: Displacement measurements based on the first category depend on the viewing angle of the camera/laser as well as the marker position. It is not easy to reproduce the same setup from day to day. Daily setup variations may result in errors in the prediction of target position. Spirometers do not suffer from these setup problems.

A spirometer was originally designed for measuring a patient's pulmonary function. This measurement extends usually over a relatively short period of time sufficient to cover a few breathing cycles. However, for breathing monitoring, stable operation of the spirometer over an extended time period is required. Due to several reasons, described in the following, the spirometer signal usually drifts away from its original baseline significantly. This impairs the ability to use a spirometer for breathing monitoring. In this study, techniques have been developed to reduce signal drift.

\section{MATERIALS AND METHODS}

\section{A. Spirometer}

In this study, a CPX Spirometer (Medgraphics, St. Paul, MN) system is used. A preVent ${ }^{\mathrm{TM}}$ Pneumotach was attached to a mouthpiece (Fig. 2). The pneumotach is a bi-directional differential pressure sensor that converts the flow into a pressure signal. This signal is then converted to a voltage, digitized to a reading value $R$, and transferred to a control computer. For laminar flow, Bernoulli's law holds:

$$
\frac{1}{2} \rho f^{2}+\Delta P=\text { const., }
$$

where $f$ is the flow, $\rho$ the air density, and $\Delta P$ the induced differential pressure. 


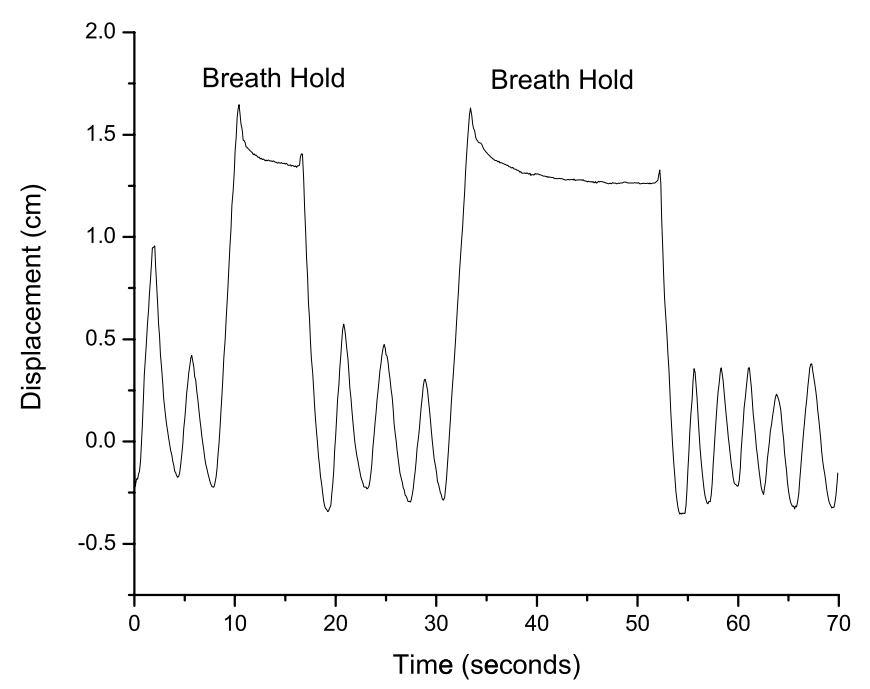

FIG. 1. Abdomen surface displacement measurement using laser-based displacement sensor during breath hold treatment. Pseudomotion happens during breath-hold due to relaxation of the abdominal muscles.

Under the assumption of the validity of Bernoulli's law the spirometer reading $R$, which is proportional to $\Delta P$, is proportional to the square of the flow, such that it can be written as

$$
R=k \cdot f^{2} \text { or } f=\sqrt{\frac{R}{k}},
$$

where $k$ is a constant. For an ideal laminar flow situation and linear pressure response sensor, spirometers based on Ber-
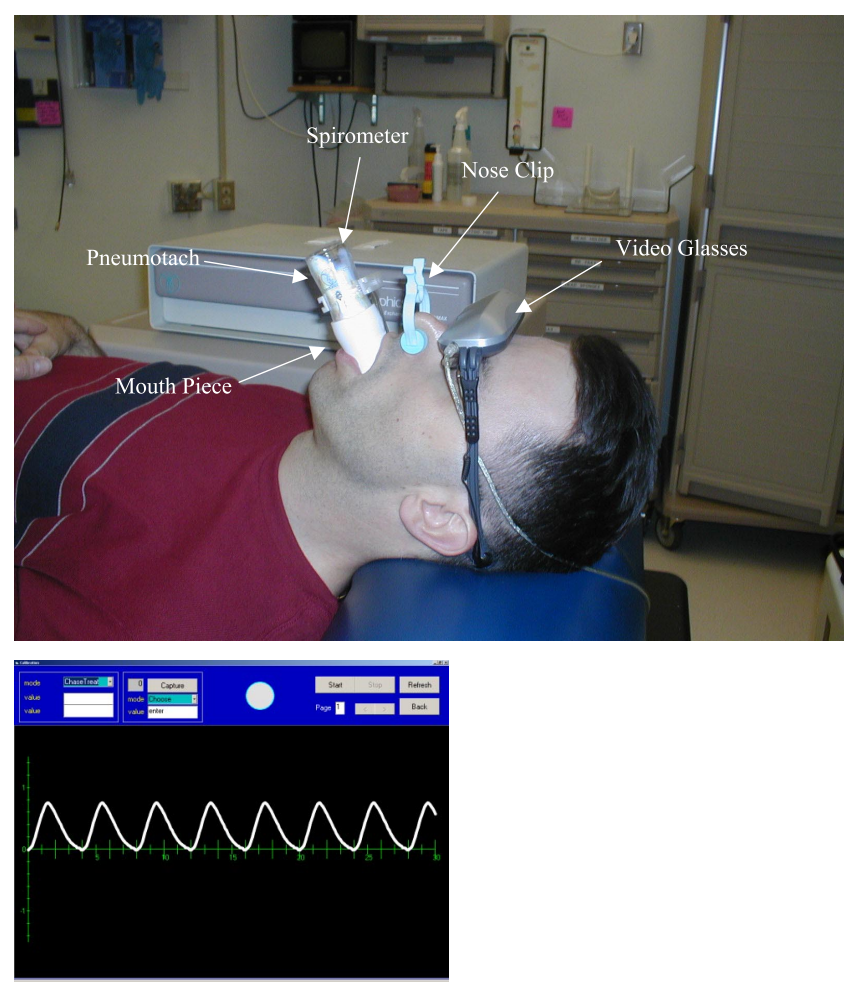

FIG. 2. The spirometer-based respiratory motion monitoring system. Video glasses are used to display the computer screen to the patient. noulli's principle, flow is expected to be proportional to $R^{1 / 2}$. In other words, such spirometers are much less sensitive to low flows than to high flows.

\section{B. Sources of air signal drift}

Drifts in the measured air volume originate from flow measurement errors. An uncertainty $\Delta f$ caused by an uncertainty $\Delta R$ in the reading leads to an uncertainty in the measured volume $\Delta V$,

$$
V+\Delta V=\int(f+\Delta f) \times d t
$$

where $V$ and $f$ are the actual air volume and flow, respectively, $\Delta V$ and $\Delta f$ are the uncertainties, and $d t$ is the time interval between two measurements or the sampling interval. In general, $\Delta f$ can be written as the sum of a systematic and a zero-mean random component. Any systematic component of $\Delta f$ causes an increase or decrease of the volume signal. For a periodic breathing pattern, $\Delta V=\int \Delta f \times d t$ is approximately constant for every cycle, thereby increasing the drift linearly with time.

The main components of the signal drift are flow sensitivity calibration error and an imperfect manual zero-flow adjustment. The first component is due to a nonlinear pressure sensor response and nonideal laminar flow, such that the spirometer response does not satisfy Eq. (2) exactly (see Sec. IIC). The second component causes a signal drift during long term operation that can be accounted for with appropriate flow baseline adjustments (see Secs. II D-II F).

Other components of signal drift are due to air turbulence, air leakage, internal pressure sensor variations, etc. Air turbulence is strongly dependent on how the airflow is channeled between mouth and spirometer. It was noted that a mouthpiece such as the one shown in Fig. 2 caused much less drift than a face mask. To further reduce air turbulence, additional filters may be added to both ends of the pneumotach. Air leakage can be prevented by carefully examining the patient connection. In a controlled environment (constant temperature, etc.) it can be assumed that the pressure sensor variations are random in nature and are therefore canceled out in the integral.

\section{Flow sensitivity calibration}

To calibrate the spirometer a $3 l$ calibration syringe (Medgraphics Corp, St. Paul, MN) was used. The mouthpiece with pneumotach was attached to the syringe. To take account of nonideal situations and also make this method valid for other types of spirometer, we assume Eq. (2) is not operative. Flow sensitivity calibration includes two steps: flow sensitivity function estimation and daily parameters update. Flow sensitivity function estimation finds the form of the flow sensitivity function and only needs to be done once, while daily parameters update is to obtain accurate parameters in flow sensitivity empirical function and in principle needs to be done before every operation.

Flow sensitivity function estimation: Airflow can be written as a function of the measurement reading as 


$$
f=C(R) \times R .
$$

The air volume pressed out or taken in for each push or pull of the piston, respectively, was $3 l$. For each piston operation, we obtained

$$
\sum_{i}^{N} C\left(R_{i}\right) R_{i} \Delta t= \pm 3 l,
$$

where the proportional constant $C\left(R_{i}\right)$ are the unknowns and are a function of flow. Each piston operation gives one equation. Theoretically, as many equations as the number of reading samples are needed to solve for $C\left(R_{i}\right)$. To reduce the number of equations needed, the flow sensitivity function is split into $n$ different domains with $C(R)=C_{m}$ for $R_{m} \leqslant R$ $\leqslant R_{m+1}(m=1, \ldots, n)$. Operating the piston (either push or pull) then results in an equation for the unknown levels $C_{m}$ :

$$
\begin{aligned}
\sum_{i}^{N} C\left(R_{i}\right) R_{i} \Delta t & \approx \sum_{m=1}^{n} C_{m} \sum_{i=1}^{k_{m}} R_{i} \Delta t \\
& \approx \sum_{m=1}^{n} k_{m} C_{m} \bar{R}_{m} \Delta t= \pm 3 l,
\end{aligned}
$$

where $N$ is the number of reading samples during one syringe operation, and $k_{m}$ is the number of readings in the $m$ th domain. As the number of levels $n$ is usually much smaller than the number of readings $N$, the number of instances $k_{m}$ of $C_{m}$ is much larger than 1 . This justifies the use of the mean reading $\bar{R}_{m}$ in Eq. (6). If at least $n$ syringe operations are performed the linear system of equations can be solved for the unknown $C_{m}$.

The resulting set of values $C_{m}$ provides an approximate solution only and is not good enough to be used directly. Nevertheless, this approach is useful in finding an empirical closed-form expression for $C(R)$ using the values $C_{m}$ as approximate initial values.

We found a simple empirical function of the form

$$
C(R)=a \times R^{b}
$$

implemented for this "Bernoulli-type" spirometer. The two unknowns $a$ and $b$ now replace the $n$ unknowns $C_{m}$ in Eq. (6). A daily calibration procedure is able to determine the parameters $a$ and $b$ with only a few syringe operations.

Daily update of the parameters: The $a$ and $b$ obtained from flow sensitivity function estimation are not accurate enough. Also because of measurement condition changes, $a$ and $b$ need to be updated frequently. After $s$ syringe operations the unknowns $a$ and $b$ for positive and negative flow can be solved by minimizing the residue $r$ defined by

$$
r=\sum_{j=1}^{s}\left(\left|\sum_{i=1}^{N_{j}} a R_{i}^{b+1} \Delta t\right|-3\right) l .
$$

The variables $N_{j}$ are the number of readings during a single syringe operation. A Nelder-Mead unconstrained optimization method, implemented in MATLAB, was used. Minimizing the absolute difference to the total syringe volume guarantees the minimization of random fluctuations during calibration. Separate optimizations have to be performed for positive and negative flow readings. The quality of the calibration procedure was tested for several simulated breathing patterns with the syringe.

\section{Dynamic volume baseline adjustment}

Many studies show that the exhale phase of normal breathing is the most reproducible phase. ${ }^{14,18}$ It is rational to choose this phase as the signal baseline. When a patient performs normal breathing, the minima of the breathing curve are recorded. However, due to irregular breathing, the minima of the breathing curve may not always be in a fully relaxed expiration. These irregular minima disturb the estimation of the baseline. Using the RANSAC (Random Sample Consensus) algorithm, ${ }^{19}$ the irregular minimum points of the volume curve are screened out. Assuming that among $n$ minimum points, there are at least $m(m<n)$ "good" points (fully relaxed expiration), the RANSAC algorithm selects randomly $m$ out of $n$ samples, calculates the standard deviation of the $m$ samples, and only accepts these if the standard deviation is smaller than a given threshold. $\Delta B$ is then the mean of these $m$ samples. In our software, $n=5$ and $m=3$ were used. Dynamic volume baseline adjustment corrects the volume signal by the magnitude of $\Delta B$ after every $n$ breathing cycles.

\section{E. Dynamic flow baseline adjustment}

Even with careful manual adjustment, there is still a residual reading left in the case of zero flow. This residual reading also tends to change during the operation period. We noticed that this can be improved by subtracting a flow correction $\Delta R_{\text {res }}$ directly from the reading. The dynamic flow baseline adjustment is performed after every dynamic volume baseline adjustment,

$$
\Delta R_{\text {res }}(k+1)=\Delta R_{\text {res }}(k)+c \times \Delta B(k),
$$

where $\Delta R_{\text {res }}$ is the residual needed to be subtracted from the reading $R, c$ is a constant that incorporates the change in units from volume to flow, and $\Delta B$ is the baseline adjustment returned by the RANSAC algorithm. $\Delta R_{\text {res }}$ is updated after every volume baseline adjustment and $k$ is the update index number. Residual $\Delta R_{\text {res }}$ converges to a fixed value after a few iterations. The convergence rate is controlled by the value of $c$.

\section{F. Zero-flow noise suppression}

For this specific spirometer, a manual flow baseline adjustment at the beginning of the operation based on a potentiometer is intended to set the reading to zero when there is no flow. However, due to electronic noise, the signal fluctuates around zero. From the empirical equation (7) it is clear that low flow signals require a large multiplicative gain, therefore the electronic noise will be amplified after correction with the flow sensitivity function. We solved this problem by forcing the readings $R_{i}$ equal to zero when they are 


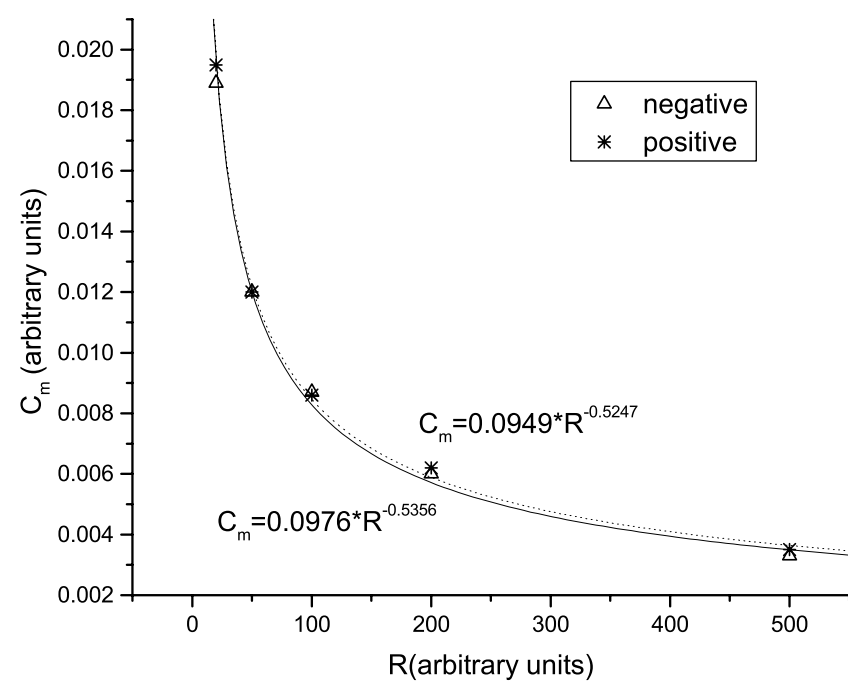

FIG. 3. Flow sensitivity function estimation for the spirometer. The five values of $C_{m}$ in Eq. (6) for positive and negative flow are plotted as stars and triangles, respectively.

smaller than a specified threshold, usually 2 to 5 digital units. In practice, this correction is only important during breath hold.

\section{RESULTS}

To establish the form of the empirical closed-form equation, a sufficient number of initial values $C_{m}$ of the function $C(R)$ were generated. Ten syringe operations were performed. Consequently, the range of readings $R$ was binned into ten domains, five for positive flow and five for negative flow. $C_{m}$ was obtained by solving the linear equations (6).

The parameters $a$ and $b$ were then determined by least square fitting of the $C_{m}$ (Fig. 3). From the figure, it can be seen that the $C_{m}$ can be fitted well by Eq. (7). Using $s=4$ syringe operations for both negative and positive flow, Fig. 4 shows the volume readings for the syringe operations before

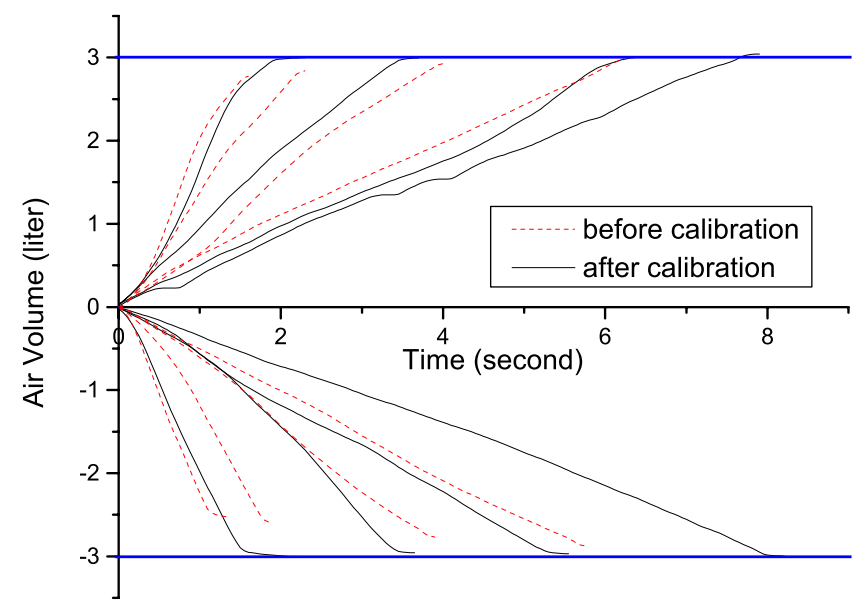

FIG. 4. Flow sensitivity function parameters calibration using $3 l$ calibration syringe. Before calibration, the spirometer signal was not able to reach \pm 3 for different flow rate. The test syringe operation after calibration shows the spirometer is able to measure air volume accurately.

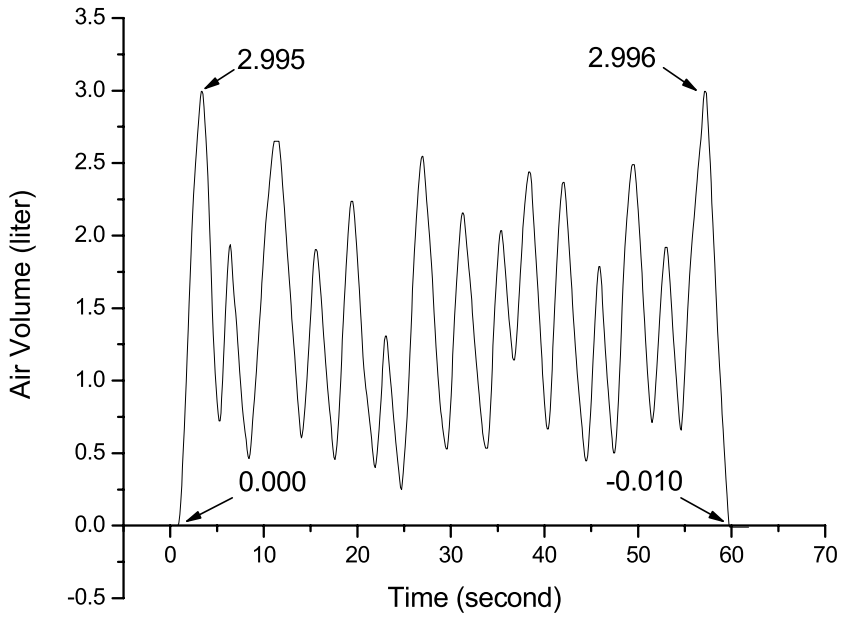

FIG. 5. Simulated breathing motion with the calibration syringe. Several "check points" show that the drift is very small after flow sensitivity calibration.

and after the calibration procedure. Before calibration, the reading is converted to a volume signal using the last stored values of $a$ and $b$ from a previous calibration. It is clearly seen that this old parameter set is not valid anymore for the current calibration. After optimization, a new set of $a$ and $b$ parameters are obtained. Figure 4 also shows the measured volume for eight test operations with the syringe using the new parameters $a$ and $b$ from the calibration. For all chosen flow rates, the final volumes were all close to $3 l$ with a variation within $1 \%$. The mean values of $a$ and $b$ for repeated calibration procedures were 0.084 and -0.493 , respectively.

The fact that $b$ is very close to -0.5 indicates that the spirometer behaves almost ideally according to Bernoulli's law. This means that the air turbulence caused by the syringe and the attached mouthpiece is small. Daily calibration can be done in under $1 \mathrm{~min}$.

The quality of the calibrated parameter set was again tested by simulating free breathing patterns with the pneumotach attached to the syringe. This way, the volume signal can be forced through well-defined control points like $V$ $=0$ for a fully pushed in piston or $V=3 l$ for fully retracted piston. Figure 5 shows such a simulated free breathing curve. Dynamic volume baseline and flow baseline adjustments were disabled in this test. After $1 \mathrm{~min}$, a control point of $V$ $=0$ was chosen for which the signal reading was $-0.01 l$. Consequently, flow sensitivity calibration alone is able to effectively reduce the signal drift.

Figure 6 shows the performance of the dynamic baseline adjustment for a spirometer measurement of a free-breathing pattern of a patient. With $c=5$ in Eq. (9) and $m=3$, and $n$ $=5$ in the RANSAC algorithm the dynamic flow baseline and volume baseline adjustments became stable after about $50 \mathrm{~s}$. Mostly due to the patient's random breathing variation, the air volume baseline fluctuated within a range of about $0.1 l$.

In our institution, the spirometer is used for deep inspiration breath-hold lung cancer treatments. So far, all patients 


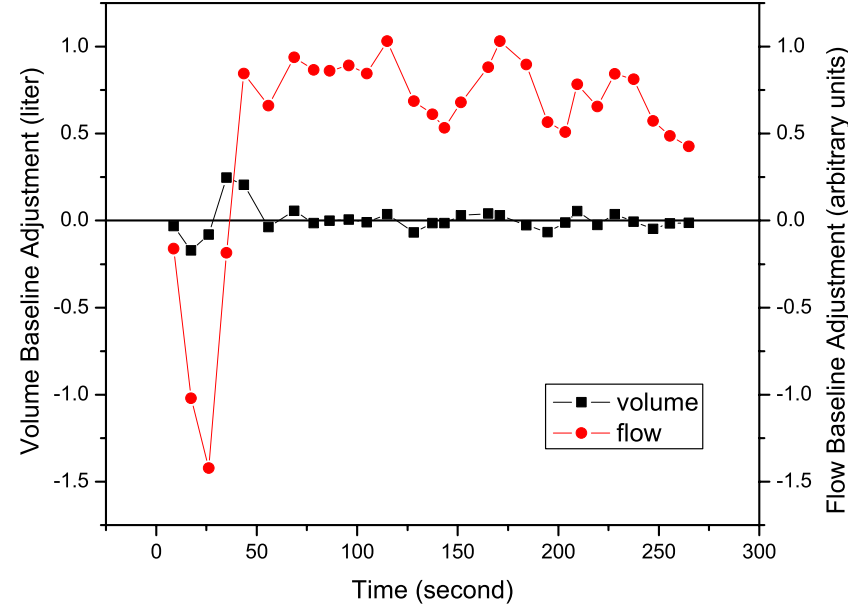

Fig. 6. Flow and volume baseline adjustments with $c=5$ in Eq. (9). Baseline adjustments become stable within $1 \mathrm{~min}$. The air volume baseline variation is within $0.1 l$, which is about $20 \%$ of normal breathing signal amplitude.

were able to tolerate the mouth piece. To guide the patient to hold breath at the same level, video glasses (Olympus FMD$250 \mathrm{~W}$ ) were used to display the breathing curve and the target level to the patient. As an example, Fig. 7 shows a patient's breathing curve before, during, and after a breath hold. Figure 7 shows that the baseline adjustments work well during the free-breathing period. The patient is usually asked to breath out forcefully before the breath hold. RANSAC is immune against the one dip before breath-hold.

\section{DISCUSSION}

In this work, several approaches were investigated to reduce the drift inherent in commercial spirometers. It was found that flow sensitivity calibration plays the most important role. It assures that the spirometer produces accurate air volume measurements and that it also reduces signal drift. Dynamic volume and flow baseline adjustments further control the drift. This study was performed on a Bernoulli-type

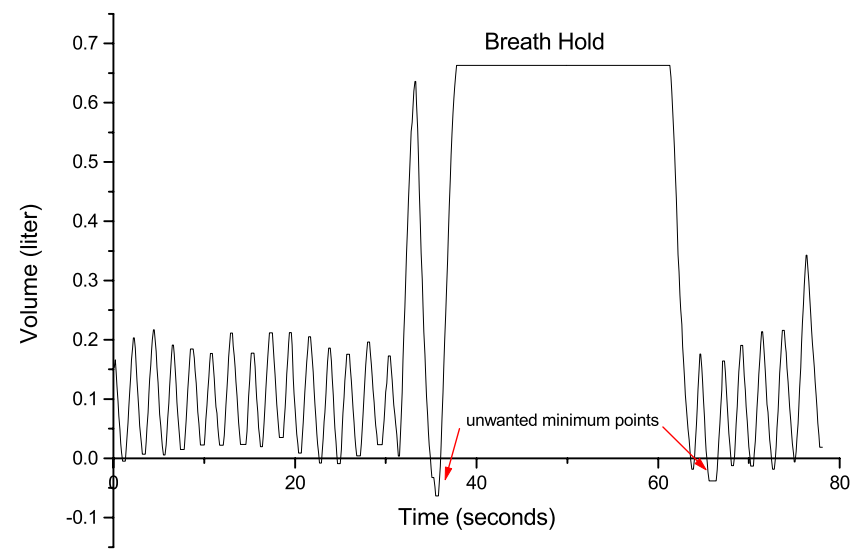

FIG. 7. Patient's breathing curve during DIBH treatment. Dynamic air volume and flow baseline adjustment further control the signal drift. Abnormal minima due to patient's irregular breathing are screened out by RANSAC algorithm. spirometer. Other spirometer types may use different types of sensors to measure airflow, and therefore require a different empirical function $C(R)$. Hence, Eq. (7) may not hold for spirometers which measure airflow not based on Bernoulli's principle. However, a very general method to calibrate flow sensitivity has been described in this work. The steps described in Sec. IIC do not depend on the particular form of the fitting function for which Eq. (7) is just an example for a Bernoulli-type spirometer.

Reverting to the Bernoulli-type spirometer described in this study and its fitting function (7) it is clear that at least two piston operations are needed to determine the two unknown parameters. Theoretically, a larger number of piston operations reduce the calibration error. However, Eq. (7) may not be adequate to cover the entire range of flow readings. If this is the case, then, during calibration, it must be assured that the flow values cover the entire range evenly. For this particular spirometer, the readings $R_{i}$ are in the range of -60 to +60 digital units during free breathing of a normal healthy person. By our experience, operating the pneumotach with the syringe tends to yield higher signal values (larger than 100 digital units) because it is not easy to pull or push the syringe slowly. However, because we found that this particular spirometer can be well characterized by Eq. (7), this is not a serious drawback.

Spirometer signal drift is well controlled with our calibration and stabilization techniques. A stable signal can be used for gating during free breathing or breath-hold treatment. There are two major components of errors using a spirometer as a target motion tracking system-calibration error and stochastic baseline variation error. The first is due to inaccuracies of the fit of the flow sensitivity function whereas the second is due to the imperfect estimation of the true baseline. After baseline adjustment the calibration error increases with signal amplitude but is generally very small (less than 1\%). The stochastic baseline variation error is mostly due to patient's breathing variation. This error can be quite large (0.1 l corresponds to about $20 \%$ of the normal breathing amplitude for an average patient). Averaging over more breathing cycles may reduce this error. However, the baseline adjustments must be done in a short period of time to control the drift. In our case, baseline adjustments were performed every five breathing cycles, corresponding to a time of about $15-20$ s.

The minima in patient's irregular breathing may not always represent fully relaxed expiration (e.g., the one breathing phase just before the breath-hold in Fig. 7). To exclude the abnormal minima from baseline determination, a RANSAC algorithm is used since it is more efficient in selecting good samples than an exhaustive computation of all the possible combinations.

In order to discuss the applicability of a spirometer to the different gating techniques and its comparison to surface displacement measurement methods we should differentiate between free-breathing gating techniques and breath-hold gating techniques. The former should also be divided into amplitude-gated and phase-gated free-breathing techniques.

Amplitude-gated free breathing: The results of this work 
show that the stochastic baseline variation can be significant compared to the amplitude of the free-breathing signal. This variation is essentially determined by the number $m$ of sample points. This number can be made much larger for surface displacement measurements as they do not suffer from drift issues. Although setup variation causes scaling error in displacement measurement, this error is small if beam-on window is set on the exhale of normal breathing cycles. Therefore, it is expected that in this case a spirometer is disadvantageous.

Phase-gated free breathing: This kind of gating is essential for displacement measurement based system due to the phase shift between abdomen surface displacement and target position. Spirometer signal has better correlation with target position thereby phase gating is not necessary. However, a spirometer should exhibit an equally well performance as a displacement measurement system for phase gating. For example breathing phases can be determined from flow versus time breathing curve which is stable for spirometer.

Gating during deep inspiration breath hold (DIBH): In gating during DIBH, the beam-on window is far from the baseline. For displacement measurements the scaling error is significant due to daily setup variation. We have shown that a spirometer can be calibrated to a high degree accuracy which enables accurate volume measurements and is not susceptible to the variation in patient setup. The stochastic baseline variation error is small compared to the signal amplitude during DIBH, therefore this error is not of importance for this application. Hence, the spirometer-based system is expected to be superior over displacement measurement-based systems for gating during DIBH.

However, the spirometer may not be suitable for some treatment techniques that require patients to breathe following a visually displayed breathing pattern. ${ }^{20,21}$ The dynamic baseline adjustment algorithm is not applicable in this situation as the minimum points of the breathing curve may not be real relaxed expiration phases. Therefore the drift cannot be accounted for in a proper way. For such cases, we developed a special combined laser/spirometer respiratory motion tracking system ${ }^{22}$ in which a spirometer is used to calibrate the displacement signal into the air volume signal, thereby eliminating the setup variations.

One fundamental problem still remains for the spirometer system as well as the displacement measurement based system. Setting the baseline to the fully relaxed exhale phase of normal breathing cycles does not guarantee stability of the target position. Lung residual volume is likely to change during the course of radiotherapy treatment. The long term correlation between anatomical structure position and external breathing signal (including spirometer and displacement measurement) is still needed to be studied in clinical trials.

\section{ACKNOWLEDGMENT}

This work was supported by a grant from the National Institute of Health (P01 CA-88960).
${ }^{a}$ Electronic mail: tiezhizhang@students.wisc.edu

${ }^{1}$ T. Tada, K. Minakuchi, T. Fujioka, M. Sakurai, M. Koda, I. Kawase, T. Nakajima, M. Nishioka, T. Tonai, and T. Kozuka, "Lung cancer: Intermittent irradiation synchronized with respiratory motion-results of a pilot study," Radiology 207, 779-783 (1998).

${ }^{2}$ H. D. Kubo, P. M. Len, S. Minohara, and H. Mostafavi, "Breathing synchronized radiotherapy program at the University of California Davis Cancer Center," Med. Phys. 27, 346-353 (2000).

${ }^{3}$ R. Wagman, E. Yorke, E. Ford, P. Giraud, G. Mageras, R. Minsky, and K. Rosenzweig, "Respiratory gating for liver tumors: Use in dose escalation," Int. J. Radiat. Oncol., Biol., Phys. 55, 659-668 (2003).

${ }^{4}$ J. Hanley, M. M. Debois, D. Mah, G. S. Mageras, A. Raben, K. Rosenzweig, B. Mychalczak, L. H. Schwartz, P. J. Gloeggler, M. T. (A.S.C.P.), W. Lutz, C. C. Ling, S. A. Leibel, Z. Fuks, and G. J. Kutcher, "Deep inspiration breath-hold technique for lung tumors: The potential value of target immobilization and reduced lung density in dose escalation," Int. J. Radiat. Oncol., Biol., Phys. 45, 603-611 (1999).

${ }^{5}$ J. W. Wong, M. B. Sharpe, D. A. Jaffray, V. R. Kini, J. M. Robertson, J. S. Stromberg, and A. A. Martinez, "The use of active breathing control $(\mathrm{ABC})$ to reduce margin for breathing motion," Int. J. Radiat. Oncol., Biol., Phys. 44, 911-919 (1999).

${ }^{6}$ L. A. Dawson, K. K. Brock, S. Kazanjian, D. Fitch, C. J. McGinn, T. S. Lawrence, R. K. Ten Haken, and J. Balter, "The reproducibility of organ position using active breathing control (ABC) during liver radiotherapy," Int. J. Radiat. Oncol., Biol., Phys. 51, 1410-1421 (2001).

${ }^{7}$ G. S. Mageras, E. Yorke, K. Rosenzweig, L. Braban, E. Keatley, E. Ford, S. A. Leibel, and C. C. Ling, "Fluoroscopic evaluation of diaphragmatic motion reduction with a respiratory gated radiotherapy system," J. Appl. Clin. Med. Phys. 2, 191-200 (2001).

${ }^{8}$ R. Wagman, E. Yorke, E. Ford, P. Giraud, G. Mageras, B. Minsky, and K. Rosenzweig, "Respiratory gating for liver tumors: Use in dose escalation,” Int. J. Radiat. Oncol., Biol., Phys. 55, 659-668 (2003).

${ }^{9}$ S. A. Nehmeh, Y. E. Eridi, C. C. Ling, K. E. Rosenzweig, O. D. Squire, L. E. Braban, E. Ford, K. Sidhu, G. S. Mageras, S. M. Larson, and J. L. Humm, "Effect of respiratory gating on reducing lung motion artifacts in PET imaging of lung cancer," Med. Phys. 29, 366-371 (2002).

${ }^{10}$ G. D. Hugo, N. Agazaryan, and T. D. Solberg, "An evaluation of gating window size, delivery method and composite field dosimetry of respiratory-gated IMRT,” Med. Phys. 29, 2517-2525 (2002).

${ }^{11}$ S. Shimizu, H. Shirato, S. Ogura, H. Akita-Dosaka, K. Kitamura, T. Nishioka, K. Kagei, M. Nishimura, and K. Miyasaka, "Detecting of lung tumor movement in real-time tumor-tracking radiotherapy," Int. J. Radiat. Oncol., Biol., Phys. 51, 304-310 (2001).

${ }^{12}$ H. Shirato, S. Shimizu, T. Kunieda, K. Kitamura, M. V. Herk, K. Kagei, T. Nishioka, S. Hashimoto, K. Fujita, H. Aoyama, K. Tsuchiya, K. Kudo, and K. Miyasaka, "Physical aspects of a real-time tumor-treacking system for gated radiotherapy," Int. J. Radiat. Oncol., Biol., Phys. 48, 11871195 (2000).

${ }^{13}$ T. Harada, H. Shirato, S. Ogura, S. Oizumi, K. Yamazaki, S. Shimizu, R. Onimaru, K. Miyasaka, M. Nishimura, and H. Dosaka-Akita, "Real-time tumor-tracking radiation therapy for lung carcinoma by the aid of insertion of a gold marker using bronchofiberscopy," Cancer 95, 1720-1727 (2002).

${ }^{14}$ Y. Seppenwoolde, H. Shirato, K. Kitamura, S. Shimizu, M. V. Herk, J. V. Lebesque, and K. Miyasaka, "Precise and real-time measurement of 3D tumor motion in lung due to breathing and heatbeat, measured during radiotherapy," Int. J. Radiat. Oncol., Biol., Phys. 53, 822-833 (2002).

${ }^{15}$ H. Shirato, S. Shimizu, K. Kitamura, T. Nishioka, K. Kagei, S. Hashimoto, H. Aoyama, T. Kunieda, N. Shinohara, H. Dosaka-Akita, and K. Miyasaka, "Four-dimensional treatment planning and fluoroscopic realtime tumor tracking radiotherapy for moving tumor," Int. J. Radiat. Oncol., Biol., Phys. 48, 435-442 (2000).

${ }^{16}$ P. G. Seiler, H. Blattmann, S. Kirsch, R. K. Muench, and C. Schilling, "A novel tracking technique for the continuous precise measurement of tumor position in conformal radiotherapy." Phys. Med. Biol. 45, N103-110 (2000).

${ }^{17}$ Q. Chen, M. S. Weinhous, F. C. Deibel, J. P. Ciezki, and R. M. Macklis, "Fluoroscopic study of tumor motion due to breathing: Facilitating precise radiation therapy for lung cancer patients," Med. Phys. 28, 18501856 (2001).

${ }^{18}$ J. M. Balter, K. L. Lam, C. J. McGinn, T. S. Lawrence, and R. K. Ten Haken, "Improvement of CT-based treatment planning models of ab- 
dominal targets using static exhale imaging," Int. J. Radiat. Oncol., Biol., Phys. 41, 939-943 (1998).

${ }^{19}$ M. A. Fischler and R. C. Bolles, "Random sample consensus: A paradigm for model fitting with applications to image analysis and automated cartography," Commun. ACM 24, 381-395 (1981).

${ }^{20}$ T. Neicu, H. Shirato, U. Seppenwoolde, and S. Jiang, "Synchronized moving aperture radiation therapy (SMART): Average tumour trajectory for lung patients," Phys. Med. Biol. 48, 587-598 (2003).
${ }^{21}$ T. Zhang, H. Keller, R. Jeraj, R. Manon, J. Welsh, R. Patel, J. D. Fenwick, M. Mehta, T. R. Mackie, and B. Paliwal, "Breathing synchronized delivery-A new technique for radiation treatment of the targets with respiratory motion," ASTRO 44th Annual meeting, Salt Lake City, 2003.

${ }^{22}$ T. Zhang, H. Keller, R. Jeraj, M. J. O’Brien, K. Sheng, T. R. Mackie, and B. Paliwal, "Lung motion tracking with a combined spirometer-laser sensor system," AAPM 44th Annual meeting, Montreal, 2002. 